\title{
Uniqueness of optimal randomized algorithms for balanced AND-OR trees
}

\author{
Fuki Ito ${ }^{1 *}$ \\ ${ }^{1}$ Tokyo Metropolitan University, 1-1 Minamiosawa, Hachioji, Tokyo 192-0397, Japan \\ ${ }^{*}$ Corresponding author: ito-fuki@ed.tmu.ac.jp
}

Received July 15, 2021, Accepted October 15, 2021

\begin{abstract}
In this paper, we investigate optimal randomized algorithms of balanced AND-OR tree evaluation, with regards to query complexity. For balanced AND-OR trees, previous studies have made a good progress on the uniqueness of eigen-distributions, i.e., optimal mixed strategies determining an input (Suzuki \& Nakamura 2012, Peng et. al. 2016). However, it has been considered that the dual problem, the uniqueness of optimal randomized algorithms, is rather difficult, and a new approach has been needed. By introducing a group-theoretical methods, we show that the uniqueness does not hold and there are uncountably many optimal randomized algorithms.
\end{abstract}

Keywords AND-OR tree, computational complexity, randomized algorithm, transposition Research Activity Group Discrete Systems

\section{Introduction}

An AND-OR tree is a tree structure in which AND nodes and OR nodes are labeled alternately [1]. In this paper we mainly consider a balanced tree: it is an ANDOR tree where every leaf has the same length from the root, and every internal node in the same level has the same number of children [2]. We may regard an ANDOR tree as a Boolean function in which each Boolean variable appears only once. For example, Fig. 1 corresponds with the Boolean function

$$
f\left(x_{1}, \ldots, x_{6}\right)=\left(x_{1} \vee x_{2}\right) \wedge\left(x_{3} \vee x_{4}\right) \wedge\left(x_{5} \vee x_{6}\right)
$$

Our main interest is to find the equilibrium points of the query cost for function computation. Suppose that an AND-OR tree $T$ is fixed and the following two-player zero-sum game is played; player A assigns truth values 0,1 to variables $x_{1}, \ldots, x_{n}$, called an assignment, and player B chooses an algorithm, which computes the value of the root node. Note that both players decide their strategies at the same time. The cost of calculation is measured by the number of each variable queried by the algorithm. Player A's goal in choosing an assignment is to make the cost as large as possible, while Player B's goal is to make it as small as possible.

Let us review some technical terms from game theory. A mixed strategy denotes a probabilistic distribution on the set of all pure strategies. If we allow mixed strategies in a two-player zero-sum game, by von Neumann's minimax theorem, the game has an equilibrium point.

Yao's minimax lemma [3] is a variant of von Neumann's minimax theorem. Roughly speaking, in the minimax value, mixed strategies are allowed only for the mini player, and in the maxmini value, mixed strategies are allowed only for the max player, and an equilibrium exists in these settings, too.

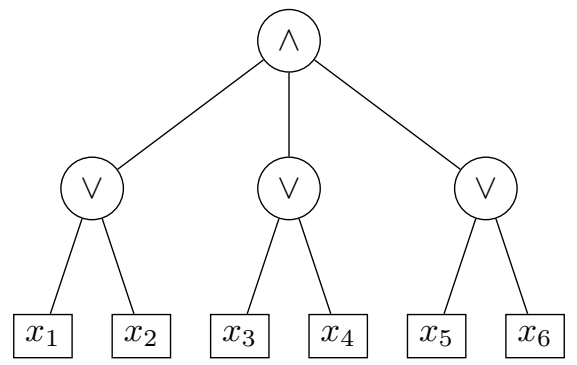

Fig. 1. A balanced AND-OR tree.

Under certain hypotheses, we may apply Yao's minimax lemma to AND-OR trees. The minimax value of the expected cost, where algorithms are randomized and assignments are not, is called the randomized complexity. We denote it by $R$. The maxmini value, where assignments are randomized and algorithms are not, is called the distributional complexity. We denote it by $P$. Based on Yao's lemma, Saks and Wigderson [4] studied the randomized complexity of an n-ary AND-OR tree, that is, a tree where every internal node has the same number of branches and every leaf has the same height (i.e. the distance from the root). They calculated the order of the randomized complexity $R$ and showed that randomized algorithms are truly superior to non-random ones in terms of worst-case cost.

In recent years, eigen-distributions of AND-OR trees have been actively studied. An eigen-distribution of an AND-OR tree is an optimal mixed strategy for max player, that is, a strategy which achieves the distributional complexity $P$. Liu and Tanaka [5] showed that eigen-distributions are unique in the case of complete binary trees. Later, Suzuki and Nakamura [6] refined Liu and Tanaka's arguments by introducing the idea of tree transposition. They also showed that when we re- 
strict the algorithms into directional ones (algorithms which query leaves in a determined order), the eigendistribution would not be unique. Peng et al. [7] showed that these results remain true in the case of balanced trees.

On the other hand, the uniqueness of optimal randomized algorithms for balanced trees was not known. Niida and Ogawa [8] applied the concept of transposition to tackle the problem, and showed several results in the case of complete binary trees. In particular, they showed (a) no-free-lunch theorem under certain conditions, and (b) that the uniform distribution on $\mathcal{A}_{\text {dir }}$ (the class of directional algorithms) achieves randomized complexity $R$.

In this paper, we will extend [8] and show that optimal randomized algorithms are not unique in the case of balanced trees. There are two points in our proof; one is to analyze the group of transpositions TR, which represents the symmetrical characteristic of balanced trees. The other is to consider randomized algorithms as elements of real vector space, in order to see that there is a small "chunk" of optimal randomized algorithms.

In Section 2, we will give definitions and introduce some previous results we will use later. In Section 3 , We will extend the results of [8] to balanced trees. In Section 4 , we will see some important properties of the group TR, and show that optimal randomized algorithms are not unique. Our main theorem is the following:

Theorem. For any balanced tree with height more than 1 , there are uncountably many randomized algorithms which are optimal with respect to $\mathcal{A}_{D}$ and $\mathcal{W}$ (which we will define in Section 2).

\section{Preliminaries}

Throughout this paper, we only consider balanced trees.

Definition 1. [9] Let $A$ be an algorithm for an AND-OR tree evaluation. $A$ is said to be depth-first if it satisfies the following: whenever $A$ queries a leaf that is a descendant of node $v$, then $A$ does not query leaves that are not descendant of $v$, unless $A$ determines the value of $v$. A depth-first algorithm $A$ is said to be an alpha-beta pruning algorithm if it omits unnecessary queries; that is, whenever $A$ finds an AND node $v$ is 0 , then $A$ never queries other descendant leaves of $v$, and similarly for OR nodes with value 1 .

By deterministic algorithm, we mean it is an alphabeta pruning algorithm.

For an AND-OR tree $T, \mathcal{A}_{D}(T)$ denotes the set of all deterministic algorithms, and $\mathcal{W}(T)$ the set of all possible assignments to $T$. In this paper we usually write $\mathcal{A}_{D}$ and $\mathcal{W}$ for simplicity. Note that these two sets are finite. For a finite set $M, \mathcal{D}(M)$ denotes the set of all probability distributions on $M$.

Definition 2. A node-code is a finite sequence of numbers that specify a node of a tree. In particular, the node-code of the root is $\varepsilon$ (empty sequence). The children of node $v$ has node-codes $v 0, \ldots, v(n-1)$, from left to right.
For example, in Fig. 1, the code of the rightmost $\vee$ is 2 , and that of $x_{5}$ is 20 .

Definition 3. Let $T$ be a tree, $A \in \mathcal{A}_{D}$ and $\omega \in \mathcal{W}$. The query history $\mathrm{qh}(A, \omega)=\left(\ell_{1}, \ldots, \ell_{k}\right)$ is the sequence of leaves queried while $A$ computes the value of $T$ with input $\omega$. The cost $C(A, \omega)$ of the computation is the length of the query history $\mathrm{qh}(A, \omega)$.

Definition 4. A randomized algorithm is an element of $\mathcal{D}\left(\mathcal{A}_{D}\right)$. For $A_{R} \in \mathcal{D}\left(\mathcal{A}_{D}\right)$ and $\omega \in \mathcal{W}$, the (expected) cost $C\left(A_{R}, \omega\right)$ is defined as $C\left(A_{R}, \omega\right)=$ $\sum_{A \in \mathcal{A}_{D}} A_{R}(A) C(A, \omega)$, where $A_{R}(A)$ is the probability of $A$ in $A_{R}$. Similarly, for $A \in \mathcal{A}_{D}$ and $d \in \mathcal{D}(\mathcal{W})$, we define $C(A, d)=\sum_{\omega \in \mathcal{W}} d(\omega) C(A, \omega)$.

Definition 5. [5] Let $T$ be a tree, $\Omega \subseteq \mathcal{W}$ and $\mathcal{A} \subseteq \mathcal{A}_{D}$.

(1) The randomized complexity with regards to $T, \mathcal{A}$ and $\Omega$ is

$$
R(T, \mathcal{A}, \Omega)=\min _{A_{R} \in \mathcal{D}(\mathcal{A})} \max _{\omega \in \Omega} C\left(A_{R}, \omega\right) .
$$

The distributional complexity with regards to $T, \mathcal{A}$ and $\Omega$ is

$$
P(T, \mathcal{A}, \Omega)=\max _{d \in \mathcal{D}(\Omega)} \min _{A \in \mathcal{A}} C(A, d) .
$$

(2) A randomized algorithm $A_{R} \in \mathcal{D}(\mathcal{A})$ is said to be optimal with respect to $(T,) \mathcal{A}$ and $\Omega$, if $\max _{\omega \in \Omega} C\left(A_{R}, \omega\right)=R(T, \mathcal{A}, \Omega)$. Similarly, a distribution $d$ on $\Omega$ is said to be an eigen-distribution with respect to $(T,) \mathcal{A}$ and $\Omega$, if $\min _{A \in \mathcal{A}} C(A, d)=P(T, \mathcal{A}, \Omega)$.

Definition 6. [6] Let $T$ be a tree, $u$ a node of $T, n$ be the number of branch of $u$, and $i \in\{1, \ldots, n-1\}$. The $i$ th $u$-transposition $\operatorname{tr}_{i}^{u}$ is defined as follows:

(a) For a node $v$ of $T$,

$$
\operatorname{tr}_{i}^{u}(v)= \begin{cases}u(i-1) w & \text { if } v=u i w \\ u i w & \text { if } v=u(i-1) w \\ v & \text { else. }\end{cases}
$$

Note that $w$ is the last half of the node-code of $v$, and $u i w$ is the concatenation of sequences $u, i$ and $w$.

(b) For an assignment $\omega \in \mathcal{W}$ and a leaf $\ell$, let $\omega(\ell)$ denote the value of assignment $\omega$ at leaf $\ell$. We define $\operatorname{tr}_{i}^{u}(\omega)(\ell)=\omega\left(\operatorname{tr}_{i}^{u}(\ell)\right)$.

(c) For a deterministic algorithm $A \in \mathcal{A}_{D}$ and an assignment $\omega \in \mathcal{W}$, if $\operatorname{qh}\left(A, \operatorname{tr}_{i}^{u}(\omega)\right)=\left(\ell_{1}, \ldots, \ell_{m}\right)$, then

$$
\mathrm{qh}\left(\operatorname{tr}_{i}^{u}(A), \omega\right)=\left(\operatorname{tr}_{i}^{u}\left(\ell_{1}\right), \ldots, \operatorname{tr}_{i}^{u}\left(\ell_{m}\right)\right) .
$$

Examples of Definition 2.6 may be found in [7].

Definition 7. [6] (1) TR $(T)$ (or simply TR) denotes the class of the composite functions of a finite number of transpositions on $T$.

(2) For $\omega \in \mathcal{W}$, the closure of $\omega$ is $\langle\omega\rangle=\{f(\omega) \mid f \in$ TR\}. We define $\langle A\rangle$ similarly for $A \in \mathcal{A}_{D}$.

(3) Let $\Omega \subseteq \mathcal{W}$ be a nonempty subset. $\Omega$ is said to be closed if for any $f \in \mathrm{TR}, f(\omega) \in \Omega$. $\Omega$ is said to be connected if for any $\omega, \omega^{\prime} \in \Omega$, there are transpositions $\operatorname{tr}_{i_{1}}^{u_{1}}, \ldots, \operatorname{tr}_{i_{k}}^{u_{k}}$ such that $\operatorname{tr}_{i_{1}}^{u_{1}} \circ \cdots \circ \operatorname{tr}_{i_{k}}^{u_{k}}(\omega)=\omega^{\prime}$, where, for any $j \in\{1, \ldots, k\}, \operatorname{tr}_{i_{j}}^{u_{j}} \circ \cdots \circ \operatorname{tr}_{i_{k}}^{u_{k}}(\omega) \in \Omega$. We define similarly for nonempty $\mathcal{A} \subseteq \mathcal{A}_{D}$.

Note that $\mathrm{TR}$ is a finite group with regards to the function composition. 
Definition 8. [5] For $i \in\{0,1\}$, the $i$-set is the set of assignments satisfying the following.

(a) The value of the root is $i$.

(b) For any AND node with value 0 , it has exactly one child of value 0 .

(c) For any OR node with value 1, it has exactly one child of value 1 .

Remark 9. Every closure is closed and connected. Conversely, every closed and connected set can be represented as the closure of its arbitrary element. The $i$-set and $\mathcal{A}_{\mathrm{dir}}$ (the set of directional algorithms) are closed and connected.

We review some previous results we will use later.

Theorem 10. (Yao's minimax lemma [3]) For any tree $T, \Omega \subseteq \mathcal{W}$ and $\mathcal{A} \subseteq \mathcal{A}_{D}$, we have $R(T, \mathcal{A}, \Omega)=$ $P(T, \mathcal{A}, \Omega)$.

Theorem 11. [7] Let $T$ be a tree. Let $\mathcal{A} \subseteq \mathcal{A}_{D}$ and $\Omega \subseteq$ $\mathcal{W}$ be both closed and connected, and $d_{\text {unif }}(\Omega)$ the uniform distribution on $\Omega$. Then $\min _{A \in \mathcal{A}} C\left(A, d_{\text {unif }}(\Omega)\right)=$ $P(T, \mathcal{A}, \Omega)$.

Theorem 12. [7] Let $T$ be a tree with AND root, $\Omega_{1}$ 1-set of $T$, and $\Omega$ a closed set of assignments which is not 1-set. Let $d_{\text {unif }}\left(\Omega_{1}\right)$ and $d_{\text {unif }}(\Omega)$ be the uniform distributions on $\Omega_{1}$ and $\Omega$, respectively. Then for any algorithm $A \in \mathcal{A}_{D}$, we have $C\left(A, d_{\text {unif }}\left(\Omega_{1}\right)\right)>C\left(A, d_{\text {unif }}(\Omega)\right)$.

\section{Extention of Niida-Ogawa Theorem}

Niida and Ogawa [8] proved the following Propositions 13, 14, Lemma 16, and Theorem 17 for binary trees. Their proof did not use properties peculiar to binary trees, except for Lemma 3.4; they showed it by means of a result in [6]. By replacing this passage by a result in [7], we can easily show these four assertions by mimicking the proofs in [8]. Note that for $f \in \mathrm{TR}$, we have $C(A, f(\omega))=C\left(f^{-1}(A), \omega\right)$.

Proposition 13. (No-free-lunch theorem) Let $\mathcal{A} \subseteq \mathcal{A}_{D}$ be connected and $\Omega \subseteq \mathcal{W}$ closed. Then there is a constant $c$ such that for any randomized algorithm $A_{R} \in$ $\mathcal{D}(\mathcal{A})$ on $\mathcal{A}$, we have $\sum_{\omega \in \Omega} C\left(A_{R}, \omega\right)=c$.

Proposition 14. Let $\mathcal{A} \subseteq \mathcal{A}_{D}$ and $\Omega \subseteq \mathcal{W}$ be both closed and connected, and $A_{R}^{u}$ the uniform distribution on $\mathcal{A}$. Then the following (a)-(d) are equivalent for any $A_{R} \in \mathcal{D}(\mathcal{A})$

(a) $A_{R}$ is optimal with respect to $\mathcal{A}$ and $\Omega$.

(b) For any $\omega \in \Omega, C\left(A_{R}, \omega\right)=c$ (constant).

(c) There is $\alpha \in \Omega$ such that $\max _{\omega \in \Omega} C\left(A_{R}, \omega\right)=$ $C\left(A_{R}^{u}, \alpha\right)$.

(d) $\max _{\omega \in \Omega} C\left(A_{R}, \omega\right)=1 /|\Omega| \sum_{\omega \in \Omega} C\left(A_{R}, \omega\right)$.

Remark 15. From this proposition, we can see that $A_{R}$ is optimal with respect to $\mathcal{A}$ and $\Omega$ if and only if $A_{R}^{u}$ and $A_{R}$ have the same cost for every assignment in $\Omega$.

Lemma 16. [7] If $\mathcal{A} \subseteq \mathcal{A}_{D}$ is closed, then $R\left(T, \mathcal{A}_{D}, \mathcal{W}\right)=R(T, \mathcal{A}, \mathcal{W})$. This implies that if $A_{R} \in$ $\mathcal{D}(\mathcal{A})$ is optimal with respect to $\mathcal{A}$ and $\mathcal{W}$, then it is also optimal with respect to $\mathcal{A}_{D}$ and $\mathcal{W}$.

Theorem 17. Suppose $\mathcal{A} \subseteq \mathcal{A}_{D}$ is closed and connected. Then the uniform distribution $A_{R}^{u}$ on $\mathcal{A}$ is optimal with respect to $\mathcal{A}_{D}$ and $\mathcal{W}$.

\section{Main Theorem}

Lemma 18. For any $\Omega \subseteq \mathcal{W}$ closed and connected, $|\mathrm{TR}| /|\Omega|$ is a positive integer which is greater than 1 .

Proof Fix $\omega \in \Omega$. Let $\mathrm{TR}_{\omega}=\{f \in \mathrm{TR} \mid f(\omega)=\omega\}$. Then we can see that $\mathrm{TR}_{\omega}$ is a subgroup of TR. In fact, for any $f, g \in \mathrm{TR}_{\omega}$, we have $f(g(\omega))=\omega, f^{-1}(\omega)=\omega$, so $g \circ f$ and $f^{-1}$ are in $\mathrm{TR}_{\omega}$. Now consider

$$
\mathrm{TR} / \mathrm{TR}_{\omega}=\left\{f_{1} \mathrm{TR}_{\omega}, \ldots, f_{m} \mathrm{TR}_{\omega}\right\} .
$$

Here $f_{i} \mathrm{TR}_{\omega}$ is a left coset, and if $i \neq j$, then $f_{i} \mathrm{TR}_{\omega} \neq$ $f_{j} \mathrm{TR}_{\omega}$. Since $\mathrm{TR}$ is finite, we can apply Lagrange theorem to see $|\Omega|=\left|\mathrm{TR} / \mathrm{TR}_{\omega}\right|=|\mathrm{TR}| /\left|\mathrm{TR}_{\omega}\right|$, which implies $|\mathrm{TR}| /|\Omega|=\left|\mathrm{TR}_{\omega}\right|$.

Now we need to prove that $\left|\mathrm{TR}_{\omega}\right|>1$, that is, $\mathrm{TR}_{\omega}$ has an element other than the identity transposition.

Case 1. Suppose $n_{h}$, the number of branches on height one (just above a leaf), is 2. If there is a height one node $u$ whose children have assignment 00 or 11 in $\omega$, then $\operatorname{tr}_{1}^{u}(\omega)=\omega$. If there is no such $u$ (e.g. $\omega=$ $0110100110 \ldots)$, then you can easily see that there must be non-identity $f \in \mathrm{TR}$ where $f(\omega)=\omega$.

Case 2. Suppose $n_{h}>2$. Then, for every height one node $u$, there are more than one 0's or 1's in their children at $\omega$, so there must be some transposition which fixes $\omega$.

(QED)

Theorem 19. (Main theorem) For any balanced tree with height more than 1 , there are uncountably many randomized algorithms which are optimal with respect to $\mathcal{A}_{D}$ and $\mathcal{W}$.

Proof First, let us show the following claim.

Claim. Let $\mathcal{A} \subseteq \mathcal{A}_{D}$ and $\Omega \subseteq \mathcal{W}$ be both connected and closed sets. Then, there are uncountably many optimal randomized algorithms with respect to $T, \mathcal{A}$, and $\Omega$.

Let $A_{R}^{u} \in \mathcal{D}(\mathcal{A})$ be the uniform distribution on $\mathcal{A}$. By Proposition 14 and Theorem 17, $A_{R}^{u}$ is optimal with respect to $\mathcal{A}$ and $\Omega$, and has exactly the same cost with any optimal $A_{R}$. In other words, if we fix $A^{*} \in \mathcal{A}$ and set $\operatorname{TR}(T)=\left\{f_{1}, \ldots, f_{k}\right\}$, we have that $A_{R}$ is optimal if and only if for any $\omega \in \Omega$,

$$
\sum_{i=1}^{k}\left[A_{R}\left(f_{i}\left(A^{*}\right)\right)-\frac{1}{|\mathcal{A}|}\right] C\left(f_{i}\left(A^{*}\right), \omega\right)=0 .
$$

Note that $A_{R}^{u}\left(f_{i}\left(A^{*}\right)\right)=1 /|\mathcal{A}|$. Now we fix $\omega^{*} \in \Omega$ and let

$M=\left(\begin{array}{ccc}C\left(f_{1}\left(A^{*}\right), f_{1}\left(\omega^{*}\right)\right) & \ldots & C\left(f_{1}\left(A^{*}\right), f_{k}\left(\omega^{*}\right)\right) \\ \vdots & \ddots & \vdots \\ C\left(f_{k}\left(A^{*}\right), f_{1}\left(\omega^{*}\right)\right) & \ldots & C\left(f_{k}\left(A^{*}\right), f_{k}\left(\omega^{*}\right)\right)\end{array}\right)$.

Consider a linear equation ${ }^{t} M x=\mathbf{0}$ where $x \in \mathbb{R}^{k}$, and let $S_{\Omega}$ be the solution space of the equation. By (1) we see that

$$
A_{R} \text { is optimal } \Leftrightarrow\left(\begin{array}{c}
A_{R}\left(f_{1}\left(A^{*}\right)\right)-\frac{1}{|\mathcal{A}|} \\
\vdots \\
A_{R}\left(f_{k}\left(A^{*}\right)\right)-\frac{1}{|\mathcal{A}|}
\end{array}\right) \in S_{\Omega} .
$$


By $\sum_{i=1}^{k}\left(A_{R}\left(f_{i}\left(A^{*}\right)\right)-1 /|\mathcal{A}|\right)=0$, the set of the optimal algorithms with respect to $\mathcal{A}$ and $\Omega$ has exactly the same cardinality as the following subset of $S_{\Omega}$ :

$$
\begin{aligned}
S_{\Omega}^{\prime}:=\left\{x \in S_{\Omega} \quad:\right. & \sum_{i=1}^{k} x_{i}=0 \\
& \text { and for any } \left.i, 0 \leq x_{i}+\frac{1}{|\mathcal{A}|} \leq 1\right\} .
\end{aligned}
$$

Informally, $S_{\Omega}^{\prime}$ is the collection of every element of $S_{\Omega}$ which represents a randomized algorithm. So it suffices to see the cardinality of $S_{\Omega}^{\prime}$. By Lemma 18, we have

$$
\operatorname{rank} M \leq\left|\left\{f_{i}(\omega): 1 \leq i \leq k\right\}\right|=|\Omega| \leq \frac{|\mathrm{TR}(T)|}{2},
$$

which implies $\operatorname{dim} S_{\Omega} \geq|\operatorname{TR}(T)| / 2$. Note that height 2 binary tree $U$ is the smallest among the trees of height more than 1 . We have $|\operatorname{TR}(U)|=8$, and since $|\operatorname{TR}(T)| \geq|\operatorname{TR}(U)|$, we have $|\operatorname{TR}(T)| / 2 \geq 4$. Thus $S_{\Omega}^{\prime}$ is uncountable. (End of the proof of the claim.)

Now, let $\Omega_{1}$ be the 1 -set and $A_{R}^{u}$ the uniform distribution on $\mathcal{A}$. Consider $S_{\Omega_{1}}^{\prime}$. By definition, we can see that $S_{\Omega_{1}}^{\prime}$ contains a line segment $\eta:[0,1] \rightarrow S_{\Omega_{1}}^{\prime}$ such that $\eta(0)=\mathbf{0}$ and $\eta(1) \neq \eta(0)$. For every $t \in[0,1]$, if we set $A_{R}^{\eta(t)} \in \mathcal{D}(\mathcal{A})$ as

$$
\left(\begin{array}{c}
A_{R}^{\eta(t)}\left(f_{1}\left(A^{*}\right)\right)-\frac{1}{|\mathcal{A}|} \\
\vdots \\
A_{R}^{\eta(t)}\left(f_{k}\left(A^{*}\right)\right)-\frac{1}{|\mathcal{A}|}
\end{array}\right)=\eta(t),
$$

then $A_{R}^{\eta(t)}$ is optimal for $\Omega_{1}$. Note that $A_{R}^{\eta(0)}=A_{R}^{u}$. Now for every $\omega \in \mathcal{W}$

$$
\begin{aligned}
\mid C & \left(A_{R}^{\eta(t)}, \omega\right)-C\left(A_{R}^{u}, \omega\right) \mid \\
& =\left|\sum_{i=1}^{k}\left(A_{R}^{\eta(t)}\left(f_{i}\left(A^{*}\right)\right)-\frac{1}{|\mathcal{A}|}\right) C\left(f_{i}\left(A^{*}\right), \omega\right)\right| \\
& =\left|\sum_{i=1}^{k}(\eta(t))_{i} C\left(f_{i}\left(A^{*}\right), \omega\right)\right| \\
& \leq\left(\max _{i}\left|(\eta(t))_{i}\right|\right) \cdot \sum_{i=1}^{k}\left|C\left(f_{i}\left(A^{*}\right), \omega\right)\right| \\
& \leq\|\eta(t)\| \cdot \sum_{i=1}^{k} C\left(f_{i}\left(A^{*}\right), \omega\right) .
\end{aligned}
$$

This implies that for any $\varepsilon>0$, there is $\delta>0$ such that

$$
\|\eta(t)\|<\delta \Rightarrow\left|C\left(A_{R}^{\eta(t)}, \omega\right)-C\left(A_{R}^{u}, \omega\right)\right|<\varepsilon .
$$

Since $\eta$ is a line segment in $\mathbb{R}^{k}$ from the origin, $\|\eta(t)\|$ is strictly increasing by $t$. Therefore,

$$
\forall \omega \in \mathcal{W} \quad \lim _{t \rightarrow 0} C\left(A_{R}^{\eta(t)}, \omega\right)=C\left(A_{R}^{u}, \omega\right) .
$$

Now let $\Omega \subseteq \mathcal{W}$ be a closed and connected set where $\Omega \neq \Omega_{1}$. By Theorem 10, we can see that

$$
\forall A \in \mathcal{A} \quad C\left(A, d_{\text {unif }}(\Omega)\right)<C\left(A, d_{\text {unif }}\left(\Omega_{1}\right)\right) .
$$

By Theorem 10, Theorem 11, Proposition 14 and Theo- rem 17 ,

$$
\forall A \in \mathcal{A} \forall \omega \in \Omega \quad C\left(A, d_{\text {unif }}(\Omega)\right)=C\left(A_{R}^{u}, \omega\right) .
$$

Note that we can replace $\Omega$ by $\Omega_{1}$ in (3). Thus we have

$$
\forall \omega \in \Omega \forall \omega_{1} \in \Omega_{1} \quad C\left(A_{R}^{u}, \omega\right)<C\left(A_{R}^{u}, \omega_{1}\right) .
$$

Since $\mathcal{W}$ is finite, by (2) and (4), we can observe the following: there is $\delta>0$ such that if $0 \leq t<\delta$,

$$
\forall \omega \in \Omega \forall \omega_{1} \in \Omega_{1} \quad C\left(A_{R}^{\eta(t)}, \omega\right)<C\left(A_{R}^{\eta(t)}, \omega_{1}\right) .
$$

Hence, if $t<\delta$, then

$$
\begin{array}{rlr}
R\left(T, \mathcal{W}, \mathcal{A}_{D}\right) & =\max _{\omega \in \mathcal{W}} C\left(A_{R}^{u}, \omega\right) & \text { (by } A_{R}^{u} \text { optimal) } \\
& =\max _{\omega_{1} \in \Omega_{1}} C\left(A_{R}^{u}, \omega_{1}\right) & \text { (by (4)) } \\
& =\max _{\omega_{1} \in \Omega_{1}} C\left(A_{R}^{\eta(t)}, \omega_{1}\right) & \text { (by Prop. 14) } \\
& =\max _{\omega \in \mathcal{W}} C\left(A_{R}^{\eta(t)}, \omega\right) & \text { (by (5)) }
\end{array}
$$

In other words, if $t$ is small enough, then $A_{R}^{\eta(t)}$ is optimal with respect to $\mathcal{A}$ and $\mathcal{W}$. Since $\eta$ is a line segment on $S_{\Omega_{1}}^{\prime}$, every $A_{R}^{\eta(t)}$ is different if $t$ is different, which implies there are uncountably many optimal algorithms with respect to $\mathcal{A}$ and $\mathcal{W}$. By Lemma 16, they are also optimal with respect to $\mathcal{A}_{D}$ and $\mathcal{W}$.

(QED)

\section{Acknowledgments}

We would like to thank Toshio Suzuki, Ryoya Kurita and Taira Shimizu for helpful discussions and writing support.

\section{References}

[1] S. Arora and B. Barak, Computational Complexity: A Modern Approach, Cambridge University Press, Cambridge, 2009.

[2] M. Tarsi, Optimal search on some game trees, J. ACM, 30 (1983), 389-394.

[3] A. Yao, Probabilistic computations: Toward a unified measure of complexity, in: SFCS 1977, pp. 222-227, IEEE Computer Society, 1977.

[4] M. Saks and A. Wigderson, Probabilistic Boolean decision trees and the complexity of evaluating game trees, in: SFCS 1986, pp. 29-38, IEEE Computer Society, 1986.

[5] C. Liu and K. Tanaka, Eigen-distribution on random assignments for game trees, Inform. Process. Lett., 104 (2007), 73-77.

[6] T. Suzuki and R. Nakamura, The eigen distribution of an AND-OR tree under directional algorithms, IAENG Int. J. Appl. Math., 42 (2012), 122-128.

[7] W. Peng, et al., The eigen-distribution for multi-branching trees, in: Proc. IMECS 2016, pp, 88-93, Newswood Limited, 2016

[8] Y. Niida and T. Ogawa, Kanzen nibun AND-OR ki jou no saiteki na rantaku algorithm ni tsuite (On optimal randomized algorithms of complete binary AND-OR trees) (in Japanese), RIMS Kôkyûroku, 1832 (2013), 158-176.

[9] D. Knuth and W. Moore, An analysis of alpha-beta pruning, Artificial Intelligence, 6 (1975), 293-326. 